Article

\title{
Implication of an Aldehyde Dehydrogenase Gene and a Phosphinothricin $N$-Acetyltransferase Gene in the Diversity of Pseudomonas cichorii Virulence
}

\author{
Masayuki Tanaka ${ }^{1}$, Ullah Md Wali ${ }^{1}$, Hitoshi Nakayashiki ${ }^{2}$, Tatsuya Fukuda ${ }^{3}$, \\ Hiroyuki Mizumoto ${ }^{1}$, Kouhei Ohnishi ${ }^{4}$, Akinori Kiba ${ }^{1}$ and Yasufumi Hikichi ${ }^{1, *}$
}

1 Laboratory of Plant Pathology and Biotechnology, Kochi University, Nankoku, Kochi 783-8502, Japan; E-Mails: tanakamasayukikani@yahoo.co.jp (M.T.); wullahsau@yahoo.com (U.M.W.); jm-mizumoto@kochi-u.ac.jp (H.M.); akiba@kochi-u.ac.jp (A.K.)

2 Laboratory of Plant Pathology, Kobe University, Kobe, Hyogo 657-8501, Japan;

E-Mail: hnakaya@kobe-u.ac.jp

3 Faculty of Agriculture, Kochi University, Nankoku 783-8502, Japan;

E-Mail: tfukuda@kochi-u.ac.jp

4 Research Institute of Molecular Genetics, Kochi University, Nankoku, Kochi 783-8502, Japan; E-Mail: kouheio@kochi-u.ac.jp

* Author to whom correspondence should be addressed; E-Mail: yhikichi@kochi-u.ac.jp; Tel.: +81-88-864-5218; Fax: +81-88-864-5200.

Received: 27 September 2011; in revised form: 28 October 2011 / Accepted: 7 November 2011 / Published: 27 December 2011

\begin{abstract}
Pseudomonas cichorii harbors the hrp genes. hrp-mutants lose their virulence on eggplant but not on lettuce. A phosphinothricin $N$-acetyltransferase gene (pat) is located between $h r p L$ and an aldehyde dehydrogenase gene $($ aldH $)$ in the genome of $P$. cichorii. Comparison of nucleotide sequences and composition of the genes among pseudomonads suggests a common ancestor of hrp and pat between $P$. cichorii strains and $P$. viridiflava strains harboring the single hrp pathogenicity island. In contrast, phylogenetic diversification of ald $H$ corresponded to species diversification amongst pseudomonads. In this study, the involvement of aldH and pat in $P$. cichorii virulence was analyzed. An aldH-deleted mutant $(\Delta$ aldH) and a pat-deleted mutant ( $\Delta$ pat) lost their virulence on eggplant but not on lettuce. $P$. cichorii expressed both genes in eggplant leaves, independent of HrpL, the transcriptional activator for the hrp. Inoculation into Asteraceae species susceptible to $P$. cichorii showed that the involvement of hrp, pat and aldH in P. cichorii virulence is
\end{abstract}


independent of each other and has no relationship with the phylogeny of Asteraceae species based on the nucleotide sequences of $n d h F$ and $r b c L$. It is thus thought that not only the hrp genes but also pat and $a l d H$ are implicated in the diversity of $P$. cichorii virulence on susceptible host plant species.

Keywords: Pseudomonas cichorii; aldH; pat; hrp; virulence; diversity

\section{Introduction}

Pseudomonas cichorii causes bacterial rot in lettuce, which is characterized by shiny, dark brown, firm necrotic spots on leaves underneath the second or the third outermost head-leaves [1-3]. $P$. cichorii reportedly causes midrib rot of greenhouse-grown butterhead lettuce [4].

In culture medium and on lettuce leaves, $P$. cichorii does not produce pectate lyase, the most important extracellular plant cell wall-degrading enzyme. The development of disease symptoms is closely associated with programmed cell death (PCD) following heterochromatin aggregation and laddering of genomic DNA in the P. cichorii-infected lettuce cells [5]. P. cichorii also causes necrotic spots on eggplant distinct from the disease symptoms on lettuce [6,7]. Kiba et al. [7] also showed that development of necrotic spot symptoms following PCD in leaves of eggplant infiltrated with $P$. cichorii was associated with de novo protein synthesis, intracellular reactive oxygen species and caspase III-like proteases.

In several Gram-negative phytopathogenic bacteria, the hrp genes ( $h r p)$ are essential determinants for disease development in compatible hosts and for elicitation of the hypersensitive response (HR) on resistant plants [8]. The hrp cluster encodes proteins of the type III secretion system (T3SS), which transports virulent proteins directly into the host cells. These proteins subsequently cause leakage of plant nutrients into the extracellular spaces of infected tissues and suppress host defenses. Nine of the hrp have been renamed $h r c$ (HR and conserved) to indicate that they encode conserved components that are also present in T3SS of the animal pathogens Yersinia, Shigella and Salmonella [9].

The hrp cluster reportedly exists in the genomic DNA of $P$. cichorii [6,10]. hrp-deficient mutants of SPC9018 grow slowly, and the appearance of disease symptoms on infected lettuce leaves is delayed compared with the wild type strain, suggesting that the putative T3SS-dependent effector proteins may hinder or delay the plant defense response, giving the bacteria time to multiply before inducing PCD in lettuce leaves [6]. On the other hand, $h r p$-deficient mutants lose both their ability to vigorously grow in eggplant leaves and their virulence on eggplant. It is thus thought that the hrp cluster may be implicated in the diversity of $P$. cichorii virulence.

Nucleotide sequences and gene composition of the hrp cluster in P. cichorii are homologous to those in the single pathogenicity island (S-PAI) of $P$. viridiflava $[6,10]$. Furthermore, Hojo et al. [6] demonstrated functional conservation of $h r p F$ operons between P. cichorii strain SPC9018 (SPC9018) and P. viridiflava strain Pv9504 (Pv9504) harboring the S-PAI. These lines of evidence suggest a common ancestor for the hrp cluster between $P$. cichorii strains and the S-PAI of P. viridiflava.

A phosphinothricin $N$-acetyltransferase gene (pat) is located between $h r p L$ and an aldehyde dehydrogenase gene $($ aldH) in genomic DNA of $P$. cichorii (Figure S1) and the BS and AS groups of 
P. viridiflava strains harboring S-PAI but not the AT group of $P$. viridiflava strains harboring the tripartite pathogenicity island (T-PAI) and P. syringae strains $[6,10,11]$. It is thought that there is a common ancestor of pat between $P$. cichorii strains and the BS and the AS group strains harboring the S-PAI in P. viridiflava [10].

Wang et al. [12] reported that aldH is involved in the virulence of Agrobacterium tumefaciens in response to starvation or host signals. Moreover, the ToxR-regulated aldH of epidemic and pandemic strains of $V$. cholerae is located at the left end of a chromosomal PAI, adjacent to a putative transposase gene which is present in epidemic and pandemic strains but absent from nonpathogenic strains [13]. The PAGI-1 region is reportedly inserted within the 3' region of aldH in genomic DNA of virulent $P$. aeruginosa strain X24509 [14]. Deduced amino acid sequences of the protein encoded by pat showed a similarity to those of phosphinothricin acetyltransferase, which is encoded by bar and specifically acetylates L-phosphinothricin that shows toxicity against microorganisms and plants, leading to resistance of the bacteria to L-phosphinothricin [15-17]. Though the available evidence suggests aldH and pat may be involved in environmental responses, we have no information on the involvement of aldH and pat in $P$. cichorii virulence. In this study, we thus analyzed the involvement of ald $H$ and pat in the diversity of $P$. cichorii virulence on susceptible host plant species.

\section{Results and Discussion}

\subsection{In Vitro Growth of the aldH-Deficient Mutant and the pat-Deficient Mutant of P. cichorii}

To compare the in vitro growth ability of the ald $H$-deficient mutant $(\Delta \mathrm{ald} H)$ and the pat-deficient mutant ( $\Delta$ pat) from SPC9018 with that of SPC9018, P. cichorii strains were incubated in PY-medium and the optical density at $600 \mathrm{~nm}$ (OD600) of the bacterial suspensions was measured. The growth rate of both $\Delta$ aldH and $\Delta$ pat was equal to that of SPC9018, showing that deletion of aldH and pat does not affect in vitro growth of the bacteria (Figure 1).

Figure 1. Growth of P. cichorii strains incubated in PY-medium.

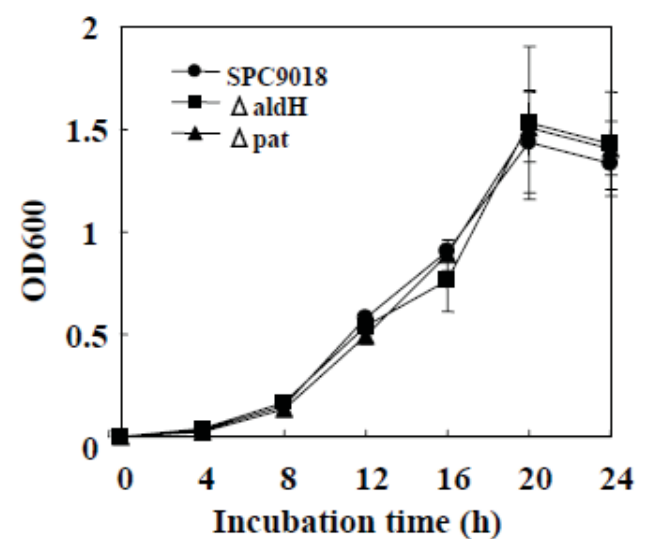

\subsection{Involvement of aldH and pat in Virulence of P. cichorii on Eggplant but not on Lettuce}

When inoculated with SPC9018, symptoms on leaves of eggplant and lettuce developed within one day post-inoculation and then progressed beyond the inoculated area within three days post-inoculation (Figure 2). The $\Delta \mathrm{aldH}$ and the $\Delta$ pat mutants lost their virulence on eggplants (Figure 2A). However, 
both mutants exhibited virulence on lettuce, similar to SPC9018 (Figure 2B). To confirm the involvement of aldH and pat in $P$. cichorii virulence against eggplant, the $\Delta$ aldH and $\Delta$ pat mutants were complemented with plasmids pPc-aldH and pPc-pat carrying aldH and pat, respectively, from the SPC9018 genome. The transformants, $\Delta$ aldH(Pc-aldH) and $\Delta$ pat(Pc-pat), exhibited virulence against eggplant similar to SPC9018 (Figure 2A). These results indicate involvement of aldH and pat in SPC9018 virulence on eggplant but not on lettuce.

Figure 2. (A) Necrotic lesions on eggplant leaves three days post-inoculation, and (B) rot on lettuce leavesone day post-inoculation with P. cichorii. Strains (a) SPC9018; (b) $\Delta$ aldH; (c) $\Delta$ aldH(Pc-aldH); (d) $\Delta$ aldH(Pv-aldH); (e) $\Delta$ aldH(Pst-aldH); (f) $\Delta$ aldH(Pa-aldH); (g) $\Delta$ pat; (h) $\Delta$ pat(Pc-pat); (i) $\Delta$ pat(Pv-pat): were inoculated at a bacterial density of $1.0 \times 10^{8} \mathrm{cfu} / \mathrm{mL}$ in distilled water in a $20 \mu \mathrm{L}$ volume (circled); Leaves were inoculated with distilled water (j) as a negative control. Plants were grown at $25^{\circ} \mathrm{C}(10,000 \mathrm{~L} \times 16 \mathrm{~h} /$ day $)$.

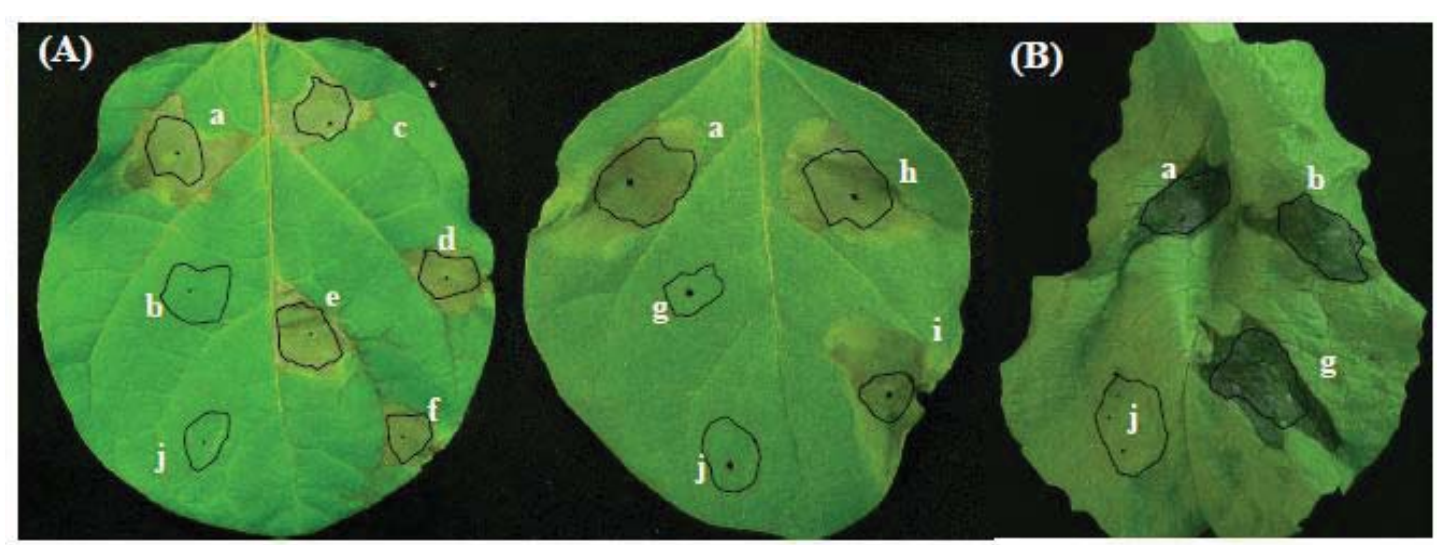

\subsection{Involvement of pat, but not aldH, in Bacterial Growth in Planta}

The $\Delta$ aldH mutant grew vigorously in the inoculated area of eggplant leaves, and reached a maximum population size of $2.6 \times 10^{7} \mathrm{cfu} / \mathrm{cm}^{2}$ at $24 \mathrm{~h}$ post-inoculation, similar to SPC9018 and the complemented strain $\Delta \mathrm{aldH}(\mathrm{Pc}$-aldH) (Figure 3). In contrast, the $\Delta$ pat mutant grew slower compared to SPC9018 and reached a maximum population size of $1.9 \times 10^{7} \mathrm{cfu} / \mathrm{cm}^{2}$ at $36 \mathrm{~h}$ post-inoculation. The complemented strain $\Delta$ pat(Pc-pat) grew similarly to SPC9018.

The deduced amino acid sequences based on the nucleotide sequences of ald $H$ and pat showed that the amino acid sequences characteristic of type III effectors [18] were not observed in the $N$-terminal of the proteins encoded in aldH and pat. The PSORT prediction of deduced amino acid sequences of the proteins encoded in ald $H$ and pat showed localization in the bacterial cytoplasm and the bacterial inner membrane, respectively. Though the PSORT prediction is limited to identify secreted proteins through the Sec pathway, it is thought that SPC9018 may not extracellularly secrete both proteins.

Results in this study indicate involvement of pat but not aldH in bacterial growth in eggplant leaves. Hojo et al. [6] indicate that vigorous growth of SPC9018 in eggplant leaves immediately after invasion may be involved in its virulence. This evidence leads us to consider that the protein encoded in pat might be implicated in the rapid growth of $P$. cichorii immediately after invasion into eggplant leaves, and may be associated with stressors or signals present in eggplant but not in lettuce. Wang et al. [12] reported that mutation of aldH in Agrobacterium tumefaciens resulted in early 
expression of the quorum sensing signal degrading enzyme, AttM. Furthermore, aldehyde dehydrogenase is reportedly a member of virulence regulons in $V$. cholerae [13]. It is thus thought that either the signal transduction through the protein encoded in ald $H$ or the protein itself might be involved in functional regulation of virulence factors such as quorum sensing, which are involved in induction of PCD in eggplant but not in lettuce. Further analysis of aldH and pat will be required for elucidation of the virulence mechanism of SPC9018, as well as genetic and functional information regarding type III effectors.

Figure 3. Population dynamics of $P$. cichorii strains in infiltrated eggplant leaves. Values represent the mean \pm standard deviation (SD) of five separate experiments. Populations of $\Delta$ aldH, $\Delta$ aldH(Pc-aldH) and $\Delta$ pat(Pc-pat) were not significantly different from the population of $P$. cichorii strain SPC9018 $(\mathrm{P}<0.05)$ by the Student's t-test.

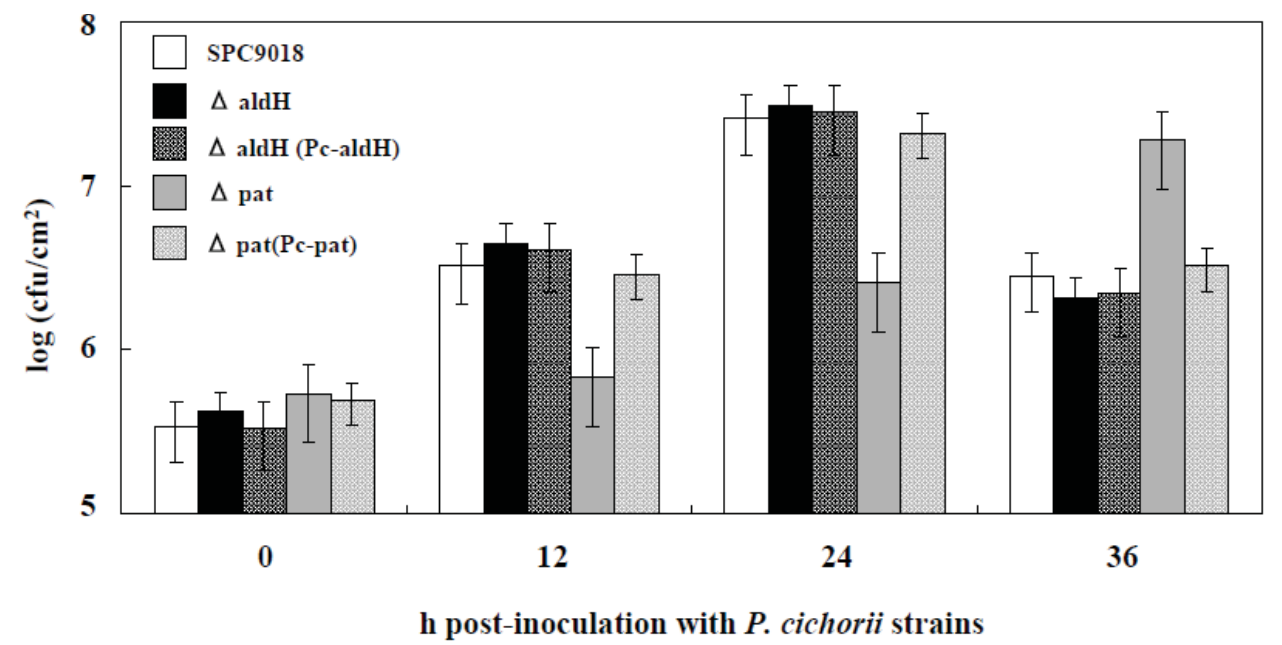

\subsection{Expression of aldH and pat is not Regulated by HrpL}

HrpL, a member of the ECF family of alternative sigma factors [19], activates the hrp, hrc and type III effector genes of pseudomonads [6,10]. To analyze the dependency of aldH and pat expression on HrpL in P. cichorii, the expression of aldH and pat was examined by RT-PCR on samples taken at $8 \mathrm{~h}$ post-inoculation. In inoculated eggplant leaves, the expression of aldH and pat in the hrpL-deleted mutant, SPC9018-L [6] was similar to the parent strain SPC9018 (Figure 4). When RNA treated with DNase I was used as template in PCR reactions, no product was observed.

Figure 4. Reverse transcription-polymerase chain reaction analysis of aldH and pat of $P$. cichorii strains infecting eggplant leaves. Total RNA was isolated from eggplant leaves at $8 \mathrm{~h}$ post-infiltration with $P$. cichorii strains.

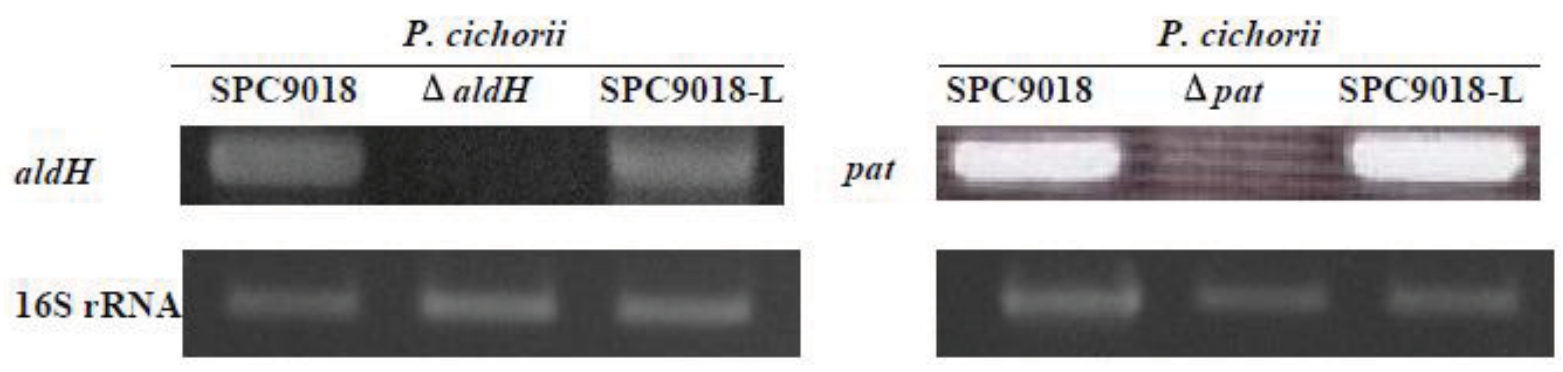


HrpL is thought to bind to a consensus bipartite cis element ( $h r p$ box) present in the promoter region of the $h r p, h r c$ and type III effector genes [19-21]. The hrp box (GGAACC-N15-16-CCANNCA) exists in the promoter regions of $h r p A, h r p F, h r p W, a v r F$, avrE and $h r p J$ in the genomic DNA of P. cichorii, and expression of these genes, is dependent on $\operatorname{HrpL}[6,10]$. Absence of the hrp box in the promoter region of aldH and pat and results in this study indicate that SPC9018 expresses aldH and pat independently of HrpL.

\subsection{Phylogenetic Diversity and Functional Conservation of aldH among Pseudomonads}

ald $H$ is also located in the genomic DNA of other pseudomonads such as $P$. viridiflava and $P$. syringae strains [10,11]. To compare the phylogenetic diversity between ald $H$, nucleotide sequences (Table S1) of aldH from $P$. cichorii, $P$. viridiflava and $P$. syringae were analyzed. In the phylogenetic tree based on the nucleotide sequence of $a l d H$, all these species represent different clusters (Figure 5).

Figure 5. Phylogenetic trees based on the nucleotide sequences of aldH. Phylogenetic trees were constructed using ClustalW (DNA Data Bank of Japan [22]) employing the Neighbor-Joining method [23]. The scale bar indicates a genetic distance, which is the expected number of substitutions per position. The numbers at the nodes represent percentage bootstrap values of 1,000 resamplings that exceeded $60 \%$. The nucleotide sequences of ald $H$ from $P$. aeruginosa strain PAO1 were used for the phylogenetic tree reconstruction.

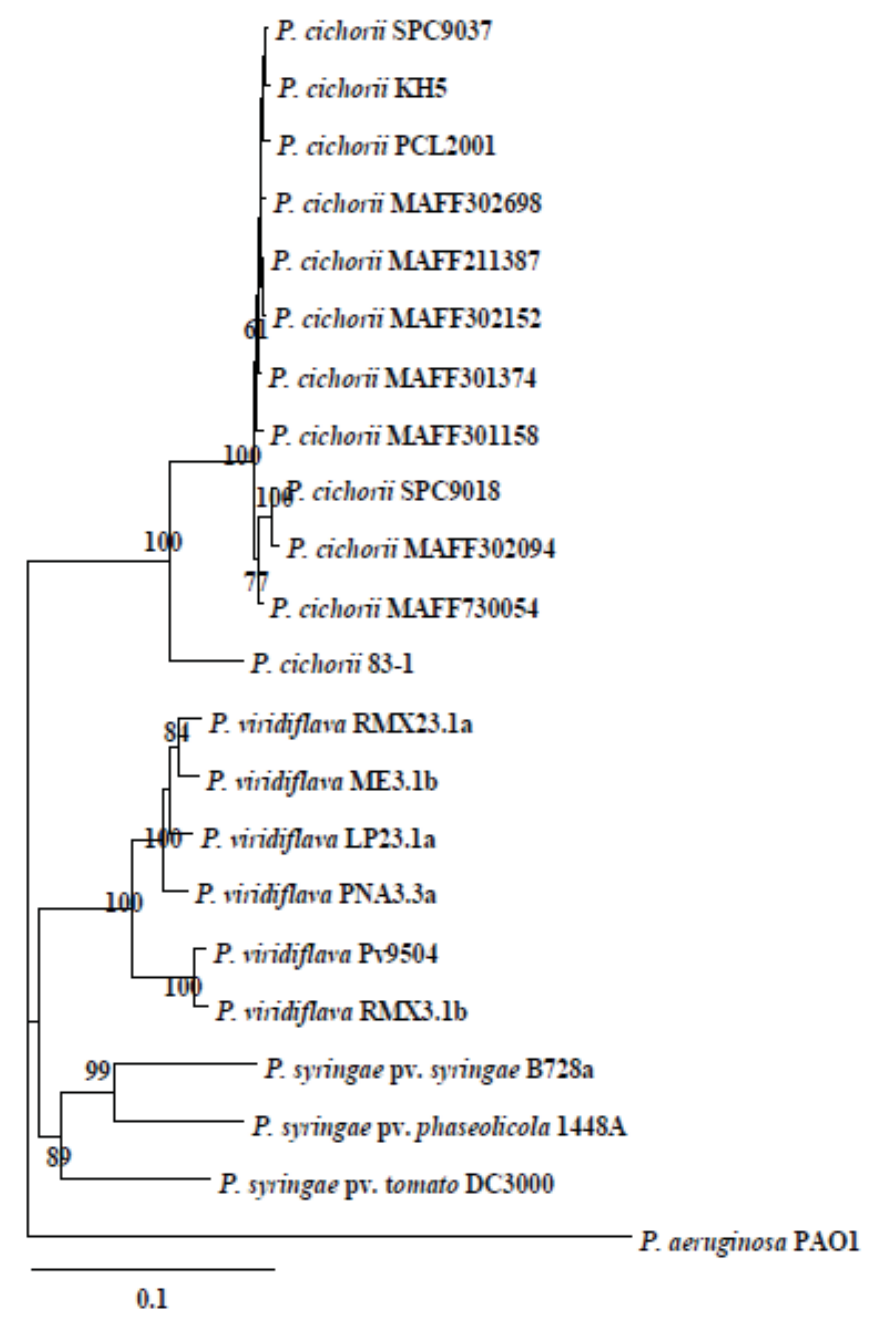


To analyze functional conservation of aldH among pseudomonads, the $\Delta$ aldH mutant was transformed with ald $H$ genes originating from the $P$. viridiflava $\mathrm{BS}$ group strain Pv9504, $P$. syringae pv. tomato strain DC3000 and $P$. aeruginosa strain PAO1, creating $\Delta$ ald $\mathrm{H}(\mathrm{Pv}$-aldH), $\Delta$ aldH(Pst-aldH) and $\Delta$ ald $\mathrm{H}(\mathrm{Pa}-\mathrm{ald} \mathrm{H})$, respectively. All transformants exhibited virulence on eggplant, similar to $\Delta$ aldH(Pc-aldH) and SPC9018 (Figure 2A). The virulence of these transformants suggests that aldH is conserved amongst pseudomonads and that phylogenetic diversification of aldH may correspond to species diversification.

\subsection{Functional Conservation of pat}

pat is located in the genome of $P$. cichorii strains and the S-PAI of $P$. viridiflava AS and BS group strains [10]. To analyze the functional conservation of pat between SPC9018 and Pv9504, the $\Delta$ pat mutant was transformed with pat originating from $P$. viridiflava strain Pv9504. The transformant, $\Delta$ pat(Pv-pat) exhibited virulence on eggplant similar to SPC9018 (Figure 2A). The virulence of this transformant suggests functional conservation of pat between $P$. cichorii and $P$. viridiflava $\mathrm{BS}$ and AS group strains harboring the S-PAI, supporting a common ancestry of pat between $P$. cichorii strains and the BS and AS group strains of P. viridiflava, as well as the hrp genes.

The composition of bacterial genomes can change rapidly and dramatically through a variety of processes, including horizontal gene transfer [24] which has been recognized as the universal event throughout bacterial evolution [14,25]. Acquiring horizontally transferred genes is an efficient way to alter the genotype of a bacterium, leading to the creation of a new phenotype or even a new species [26,27]. Although a single gene might have a low horizontal transfer index (HTI) purely by chance, it is unlikely that a large cluster of neighboring genes would all have low HTIs by chance. Such clusters are considered to be a single unit simultaneously inserted into the genome [28]. In particular, it has been suggested that a number of pathogenicity genes were horizontally transferred as large clusters, PAIs [29]. It is thus thought that a pathogenicity island in SPC9018 consists of the hrp and pat. The pathogenicity island with aldH is implicated in virulence of SPC9018 on eggplant but not on lettuce.

\subsection{Implication of hrp, aldH and pat in SPC9018 Virulence on Asteraceae Plants}

Virulence analysis showed that deletion of aldH and pat resulted in loss of SPC9018 virulence on eggplants but not on lettuce. When 31 species of Asteraceae plants (Table S2) including lettuce (Lactuca sativa) were inoculated with SPC9018, we could observe necrotic regions on leaves of all plant species at three days post-inoculation, indicating that SPC9018 is virulent on all Asteraceae plants. To analyze the involvement of $h r p$, aldH and pat in SPC9018 virulence on the Asteraceae species, we inoculated the Asteraceae plants with a $h r c C$-mutant, SPC9018-hrcC, and with the $\Delta$ aldH and $\Delta$ pat mutants. The mutants, SPC9018-hrcC, $\Delta$ aldH and $\Delta$ pat lost their virulence on 9, 16 and 6 species, respectively (Figure 6 and Table S2). Between them, all mutants lost their virulence on 5 species.

To determine the relationship between the involvement of hrp, aldH and pat in P. cichorii virulence on these plants and phylogeny among the Asteraceae species, phylogenetic trees were constructed based on the combined nucleotide sequences of $n d h F$ and $r b c L$ (Figure 6 and Table S2). The NJ phylogenetic tree placed 31 species in two major clusters (Figure 6). Involvement of hrp, aldH and pat in SPC9018 virulence on the respective species had no relationship with phylogeny of the Asteraceae 
species. It is thus thought that the implication of not only hrp but also aldH and pat in P. cichorii virulence might have arisen after species diversification of Asteraceae plants and be responsible for the virulence of $P$. cichorii towards respective species.

Figure 6. Relationship between the phylogenetic tree of Asteraceae plants based on the combined partial nucleotide sequences of $n d h F$ and $r b c L$ and virulence of SPC9018-hrcC (A), $\Delta$ aldH (B) and $\Delta$ pat (C) on Asteraceae plants. The phylogenetic tree was constructed with Clustalw using the NJ method [22,23]. The scale bar indicates genetic distance, which is the expected number of substitutions per position. $P$. cichorii strains showed virulence and non-virulence on plants with black-colored and red-colored letters, respectively. The nucleotide sequences of the combined partial nucleotide sequences of $n d h F$ and $r b c L$ from eggplant (Solanum melongena) were used as the outgroup for phylogenetic tree reconstructions.

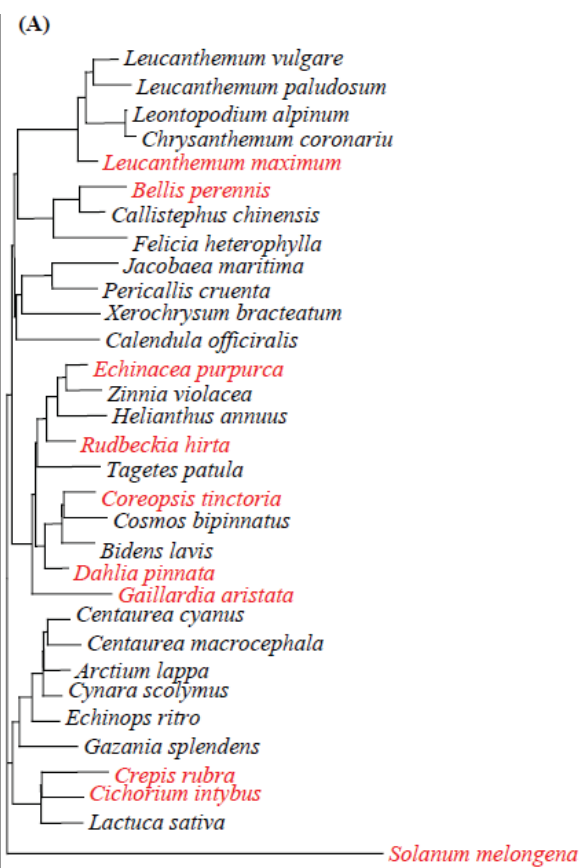

(B)

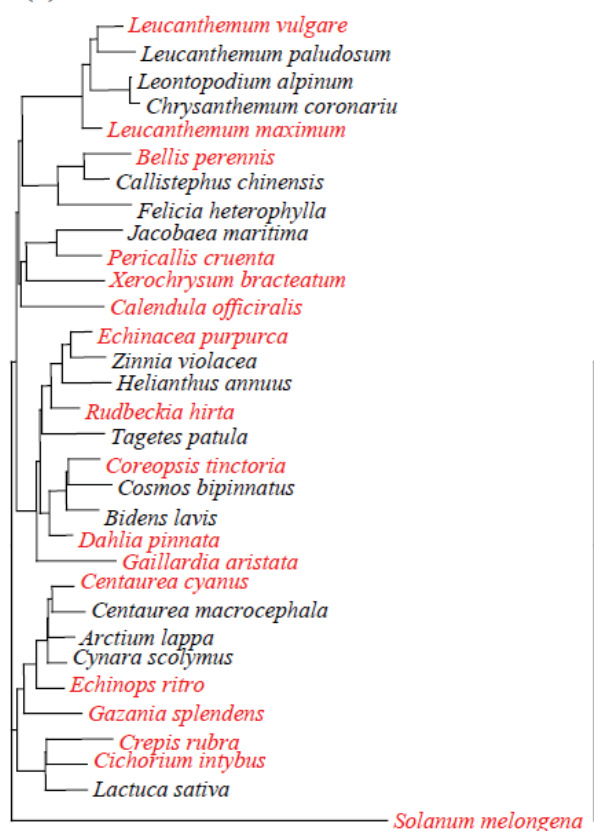

(C)

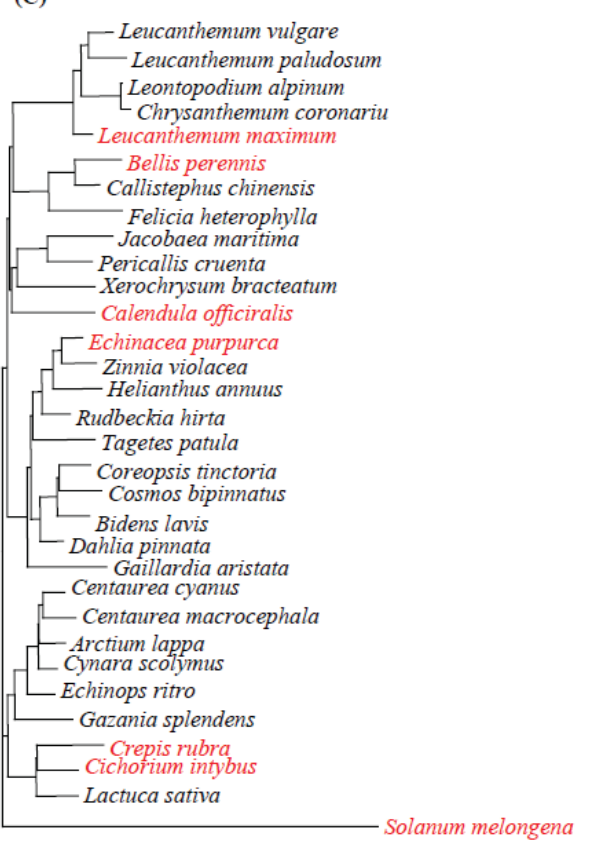

$\underline{0.01}$

Araki et al. [10] and Hojo et al. [6] demonstrated that P. cichorii strains acquired the hrp genes through horizontal transfer from a common ancestor with the S-PAI of $P$. viridiflava and implicated the hrp in its virulence. Symptoms on P. cichorii-infected lettuce leaves are characterized by shiny, dark brown, firm necrotic spots [2,3]. P. cichorii also causes necrotic spots on eggplant distinct from the disease symptoms on lettuce leaves [6,7]. The development of disease symptoms on both lettuce leaves and eggplant leaves is closely associated with PCD in P. cichorii-infected tissues [5,7]. Virulence of $P$. cichorii on eggplant is hrp-dependent. On the other hand, the hrp are not directly implicated in induction of PCD in infected lettuce leaves and the disease development on lettuce leaves caused by the hrp-deficient mutants is delayed compared with that caused by the parent strain, since the hrp genes play a role in P. cichorii growth at the early infection stages in lettuce leaves [6]. From these evidences, Hojo et al. [6] demonstrate that the effect of the hrp on P. cichorii virulence differs between lettuce and eggplant. Results in this study suggested conservation of aldH amongst pseudomonads and supported a common ancestor of pat between $P$. cichorii and $P$. viridiflava strains 
harboring the S-PAI. Furthermore, results in this study implicate not only the hrp genes but also aldH and pat in the diversity of its virulence towards susceptible host plants. Therefore, it is hypothesized that $P$. cichorii might maintain the hrp, aldH and pat to establish its virulence on respective host plants.

\section{Experimental Section}

\subsection{Bacterial Strains, Plasmids and Culture Conditions}

The bacterial strains and plasmids used in this study are listed in Table S3. P. cichorii, $P$. viridiflava, $P$. syringae pv. tomato and $P$. aeruginosa strains were routinely grown in PY-medium (5\% polypeptone, $2 \%$ yeast extract) at $30{ }^{\circ} \mathrm{C}$. Escherichia coli strains were grown in LM medium [30] at $37{ }^{\circ} \mathrm{C}$. The optimal density at $600 \mathrm{~nm}$ (OD600) of the bacterial suspensions was measured with Ultrospec 1100pro (GE Healthcare, Tokyo, Japan). Ampicillin $(50 \mu \mathrm{g} / \mathrm{mL}$, nakalai tesque, Kyoto, Japan), chloramphenicol (50 $\mu \mathrm{g} / \mathrm{mL}$, nakalai tesque), kanamycin $(50 \mu \mathrm{g} / \mathrm{mL}$, nakalai tesque) and tetracycline ( $30 \mu \mathrm{g} / \mathrm{mL}$, nakalai tesque) were used in selective media. Populations of SPC9018 and mutants in planta were assayed in three independent experiments using PCSM plates [31] and PCSM plates containing appropriate antibiotics.

\subsection{DNA Manipulations}

Isolation of genomic DNA, plasmid DNA manipulations, and PCR analysis were performed using standard techniques [6,32]. P. cichorii was transformed by electroporation as described by Hojo et al. [6]. Double-stranded DNA sequencing templates were prepared with GenElute ${ }^{\mathrm{TM}}$ Plasmid Miniprep Kits (SIGMA Chemical, St. Louis, MO, USA). Sequences were determined using an ABI Prism 3100-Avant Genetics Analyzer (Applied Biosystems, Tokyo, Japan). DNA sequence data were analyzed using the DNASIS-Mac software (Hitachi Software Engineering, Yokohama, Japan). Enzymes including restriction endonucleases (Takara, Ohtsu, Japan) and Ex-taq polymerase (Takara) were used according to the manufacturer's instructions. The primers used in this study are listed in Table S4.

\subsection{Creation of aldH- and pat-Deleted Mutants}

Plasmids designed to create deletion mutants using the kanamycin-resistant gene originating from pUCK191 [33] were based on pHSG398 (Takara) ligated sacB originating from pUCD800 [34]. The construction of the clones is described in detail in Supplementary Materials. The resulting plasmids, pD4-1-2KmSacB and pD5-1-2KmSacB, were electroporated into SPC9018 cells and the resultant kanamycin- and sucrose-resistant, and chloramphenicol-sensitive recombinants, an aldH-deficient mutant $(\Delta \mathrm{aldH})$ and a pat-deficient mutant ( $\Delta$ pat), were selected, respectively.

\subsection{Creation of the hrcC-Mutant}

For creation of the $h r c C$-mutant, a plasmid, pHSG398sacBhrcCKm, was created. The construction of the clones is described in detail in Supplementary Materials. The plasmid pHSG398sacBhrcCKm was electroporated into SPC9018 cells and a resultant kanamycin and sucrose-resistant recombinant, SPC9018-hrcC, was selected. 


\subsection{Complementation of $\triangle$ ald $H$ with aldH Originating from Pseudomonads}

For complementation of $\Delta \mathrm{aldH}$ with ald $H$ originating from pseudomonads, the plasmids, $\mathrm{pPc}$-aldH, pPst-aldH and $\mathrm{pPa}-\mathrm{aldH}$, were created. A detailed cloning procedure is described in Supplementary Materials. The plasmids pPc-aldH, pPst-aldH and pPa-aldH containing aldH, originating from SPC9018, P. syringae pv. tomato strain DC3000 and P. aeruginosa strain PAO1, were transformed into $\Delta$ ald competent cells and chloramphenicol-resistant transformants, $\Delta$ ald $\mathrm{H}(\mathrm{Pc}-\mathrm{aldH}), \Delta$ ald $\mathrm{H}(\mathrm{Pst}-\mathrm{aldH})$ and $\Delta$ aldH $(\mathrm{Pa}$-aldH) were created, respectively. The plasmid $\mathrm{pPv}$-aldH was also transformed into $\Delta$ aldH competent cells to create a tetracycline-resistant $\Delta \mathrm{aldH}(\mathrm{Pv}$-ald $\mathrm{H})$ transformant.

\subsection{Complementation of $\triangle p a t$ with pat Originating from SPC9018 and Pv9504}

For complementation of $\Delta$ pat with pat originating from SPC9018 and Pv9504, the plasmids, pPc-pat and pPv-pat, were created, respectively. A detailed cloning procedure is described in Supplementary Materials. The plasmids pPc-pat and pPv-pat including pat, originating from SPC9018 and Pv9504, were transformed into $\Delta$ pat competent cells and a chloramphenicol-resistant $\Delta$ pat(Pc-pat) transformant and a chloramphenicol-resistant $\Delta$ pat(Pv-pat) transformant, were created, respectively.

\subsection{Expression Analysis of aldH and pat in P. cichorii Strains by RT-PCR}

To analyze the expression of aldH and pat by reverse transcription-PCR (RT-PCR), total RNA was isolated from five of each set of the bacteria-infiltrated area in eggplant leaves $(0.6 \mathrm{~g}) 8 \mathrm{~h}$ after infiltration with with $50 \mu \mathrm{L}$ of the bacterial suspension $\left(1.0 \times 10^{8} \mathrm{cfu} / \mathrm{mL}\right)$ of $P$. cichorii strains, and DNase I (Applied Biosystems, Tokyo, Japan) treatment was used to remove the genomic DNA from the RNA preparation [35]. The cDNA of aldH and pat was synthesized from total RNA (6 $\mu \mathrm{g}$ ) using reverse transcription RAV-2 (Takara) utilizing primers SEMI-Back and ORF5-RT-Rv, respectively. The PCR was carried out with the primers: SEMI-Front and SEMI-Back to amplify a 500 bp amplicon specific to aldH; and ORF5-RT-Fw and ORF5-RT-Rv to amplify a $450 \mathrm{bp}$ DNA fragment specific to pat. The cDNA of $16 \mathrm{~S}$ rRNA was synthesized utilizing a primer, 16S-rRNA-Rv. The PCR was carried out with primers $16 \mathrm{~S}-\mathrm{rRNA}-\mathrm{Fw}$ and $16 \mathrm{~S}-\mathrm{rRNA}-\mathrm{Rv}$ to amplify a $448 \mathrm{bp}$ amplicon specific to $16 \mathrm{~S}$ rRNA.

\subsection{Sequencing of aldH from P. cichorii Strains}

A $1.7 \mathrm{~kb}$ DNA fragment containing aldH was amplified from the genomic DNA of $P$. cichorii strains (Table S2) and sequenced with aldH-Fw1 and aldH-Rv as primers.

\subsection{Sequencing of $\mathrm{ndh} F$ and $\mathrm{rbcL}$ from Asteraceae Plants}

The partial $n d h F$ and $r b c L$ fragments were amplified by PCR from the genomic DNA of 31 species of Asteraceae plants (Table S2) using the primers ndhF-11FW and ndhF-22RV for $n d h F$; and $1-1$ and NN3-2 for $r b c L$. The nucleotide sequences of partial $n d h F$ and $r b c L$ DNA fragments were determined directly from the PCR fragments using ndhF-11FW, ndhF-22RV, ndhF-11RV, ndhF-22FW, 1-2FW, 1-2RV, 1-1 and NN3-2. 


\subsection{Data Analysis}

The nucleotide sequences of aldH (1,584 bp), and combined $n d h F(1,996-2,023 \mathrm{bp})$ and $r b c L$ (1244-1247 bp) were aligned and phylogenetic trees were constructed using ClustalW by the neighbor-joining (NJ) method [22,23]. Genetic distances were computed with Kimura's two-parameter model [39]. The NJ phylogenetic tree was drawn by TreeView.

\subsection{Virulence Assays}

Eggplant (Solanum melongena L. cv. Senryo No. 2) and the plants listed in Table S2, including lettuce (Lactuca sativa L. cv. Success) were grown in pots containing a high-grade potting mix (Tsuchitaro; Sumitomo Forestry Co. Ltd., Tokyo, Japan) at $25{ }^{\circ} \mathrm{C}$. Light (16 h/day) was supplied at $10,000 \mathrm{~L} \times$ throughout the experimental period. Five-week-old test plants were inoculated by leaf-infiltration using a $1 \mathrm{~mL}$ disposable syringe with $1.0 \times 10^{8} \mathrm{cfu} / \mathrm{mL}$ bacteria in a $20 \mu \mathrm{L}$ volume. Following inoculation, plants were incubated for 3 days under $100 \%$ relative humidity at $25{ }^{\circ} \mathrm{C}$ and then grown at $25^{\circ} \mathrm{C}$. For all assays, inoculum concentrations were determined spectrophotometrically and confirmed by dilution plating. Plants were inspected for symptoms daily for five days after inoculation. We replicated virulence assays on tested species at five trials. Within each trial, 12 plants for each strain were treated, yielding 60 plants per strain.

\subsection{Bacterial Population in Planta}

Areas $\left(1 \mathrm{~cm}^{2}\right)$ inoculated with $P$. cichorii strains were excised from the eggplant leaves of five plants at 0,12, 24 and $36 \mathrm{~h}$ post-inoculation and ground using a mortar and pestle. Samples $(0.1 \mathrm{~mL})$ of the original solution and 10-fold serial dilutions were spread onto three plates of selective agar media containing appropriate antibiotics. Colonies were counted after 2 days of incubation at $30{ }^{\circ} \mathrm{C}$ to estimate the population.

\subsection{Nucleotide Sequence Accession Numbers}

The nucleotide sequences determined in this study have been deposited in the DDBJ/GenBank international nucleotide sequence database under the accession numbers shown in Tables S1 and S5.

\section{Conclusions}

It is thought that not only the hrp genes but also pat and aldH are implicated in the diversity of $P$. cichorii virulence on susceptible host plant species.

\section{Acknowledgments}

We thank Gregory B. Martin and Takashi Shirakawa for their kind gifts of bacterial strains. We also thank Ayami Kanda-Hojo for her critical reading. This work was supported by a Grant-in-Aid for Scientific Research awarded to Y.H. (20380029), A.K. (21580057), and K.O. (22580052) from the Ministry of Education, Science, Sports and Culture, Japan. 


\section{References}

1. Grogan, R.G.; Misaghi, I.J.; Kimble, K.A.; Greathead, A.S.; Ririe, D.; Bardin, R. Varnish spot, a destructive disease of lettuce in California caused by Pseudomonas cichorii. Phytopathology 1977, 67, 957-960.

2. Hikichi, Y.; Saito, A.; Suzuki, K. Infection sites of Pseudomonas cichorii into head leaf of lettuce. Ann. Phytopathol. Soc. Jpn. 1996, 62, 125-129.

3. Hikichi, Y.; Saito, A.; Suzuki, K. Relationship between population dynamics of Pseudomonas cichorii on lettuce and disease incidence of bacterial rot of lettuce. Ann. Phytopathol. Soc. Jpn. 1996, 62, 141-146.

4. Cottyn, B.; Heylen, K.; Heyrman, J.; Vanhouteghem, K.; Pauwelyn, E.; ABleyaert, P.; van Vaerenbergh, J.; Hofte, M.; de Vos, P.; Maes, M. Pseudomonas cichorii as the causal agent of midrib rot, anemerging disease of greenhouse-grown butterhead lettuce in Flanders. Syst. Appl. Microbiol. 2009, 32, 211-225.

5. Kiba, A.; Sangawa, Y.; Ohnishi, K.; Yao, N.; Park, P.; Nakayashiki, H.; Tosa, Y.; Mayama, S.; Hikichi, Y. Induction of apoptotic cell death leads to the development of bacterial rot caused by Pseudomonas cichorii. Mol. Plant Microbe Interact. 2006, 19, 112-122.

6. Hojo, H.; Koyanagi, M.; Tanaka, M.; Kajihara, S.; Ohnishi, K.; Kiba, A.; Hikichi, Y. The hrp genes of Pseudomonas cichorii are essential for pathogenicity on aubergine plants but not on lettuce. Microbiology 2008, 154, 2920-2928.

7. Kiba, A.; Takata, O.; Ohnishi, K.; Hikichi, Y. Comparative analysis of induction pattern of programmed cell death and defense-related responses during hypersensitive cell death and development of bacterial necrotic leaf spots in aubergine plants. Planta 2006, 224, 981-994.

8. Alfano, J.R.; Collmer, A. The type III (Hrp) secretion pathway of plant pathogenic bacteria: Trafficking harpins, avr proteins, and death. J. Bacteriol. 1997, 179, 5655-5662.

9. Bogdanove, A.J.; Beer, S.V.; Bonas, U.; Boucher, C.A.; Collmer, A.; Coplin, D.L.; Cornelis, G.R.; Huang, H.C.; Hutcheson, S.W.; Panopoulos, N.J.; et al. Unified nomenclature for broadly conserved hrp genes of phytopathogenic bacteria. Mol. Microbiol. 1996, 20, 681-683.

10. Araki, H.; Tian, D.; Goss, E.M.; Jakob, K.; Halldorsdottir, S.S.; Kreitman, M.; Bergelson, J. Presence/absence polymorphism for alternative pathogenicity islands in Pseudomonas viridiflava, a pathogen of Arabidopsis. Proc. Natl. Acad. Sci. USA 2006, 103, 5887-5892.

11. Buell, C.R.; Joardar, V.; Lindeberg, M.; Selengut, J.; Paulsen, I.T.; Lindeberg, M.; Selengut, J.; Paulsen, I.T.; Gwinn, M.L.; Dodson, R.J.; et al. The complete genome sequence of the Arabidopsis and tomato pathogen Pseudomonas syringae pv. tomato DC3000. Proc. Natl. Acad. Sci. USA 2003, 100, 10181-10186.

12. Wang, C.; Zhang, H.B.; Wang, L.H.: Zhang, L.H. Succinic semialdehyde couples stress response to quorum-sensing signal decay in Agrobacterium tumefaciens. Mol. Microbiol. 2006, 62, 45-56.

13. Parsot, C.; Mekalanos, J.J. Expression of the Vibrio cholerae gene encoding aldehyde dehydrogenase is under control of ToxR, the cholera toxin transcriptional activator. J. Bacteriol. 1991, 173, 2842-2851.

14. Liang, X.; Pham, X.Q.; Olson, M.V.; Lory, S. Identification of a genomic island present in the majority of pathogenic isolates of Pseudomonas aeruginosa. J. Bacteriol. 2001, 183, 843-853. 
15. Lutz, A.K.; Knapp, E.J.; Maliga, P. Expression of bar in the plastid genome confers herbicide resistance. Plant Physiol. 2001, 125, 1585-1590.

16. Thompson, J.C.; Movva, N.R.; Tizard, R.; Crameri, R.; Davies, E.J.; Lauwereys, M.; Botterman, J. Characterization of the herbicide-resistance gene bar from Streptomyces hygroscopics. EMBO J. 1987, 6, 2519-2523.

17. Yun, C.S.; Hasegawa, H.; Nanamiya, H. Novel bacterial $N$-acetyltransferase gene for herbicide detoxification in land plants and selection marker in plant transformation. Biosci. Biotechnol. Biochem. 2009, 73, 1000-1006.

18. Schechter, L.M.; Roberts, K.A.; Jamir, Y.; Alfano, J.R.; Collmer, A. Pseudomonas syringae type III secretion system targeting signals and novel effectors studied with a Cya translocation reporter. J. Bacteriol. 2004, 186, 543-555.

19. Xiao, Y.; Hutcheson, S.W. A single promoter sequence recognized by a newly identified alternate sigma factor directs expression of pathogenicity and host range determinants in Pseudomonas syringae. J. Bacteriol. 1994, 176, 3089-3091.

20. Innes, R.W.; Bent, A.F.; Kunkel, B.N.; Bisgrove, S.R.; Staskawicz, B.J. Molecular analysis of avirulence gene avrRpt 2 and identification of a putative regulatory sequence common to all known Pseudomonas syringae avirulence genes. J. Bacteriol. 1993, 175, 4859-4869.

21. Shen, H.; Keen, N.T. Characterization of the promoter of avirulence gene D from Pseudomonas syringae pv. tomato. J. Bacteriol. 1993, 175, 5916-5924.

22. ClustalW. Available online: http://clustalw.ddbj.nig.ac.jp/top-j.html/ (accessed on 28 October 2011).

23. Saitou, N.; Nei, M. The neighbor-joining method: A new method for reconstructing phylogenetic trees. Mol. Biol. Evol. 1987, 4, 406-425.

24. Hacker, J.; Carniel, E. Ecological fitness, genomic islands and bacterial pathogenicity. A Darwinian view of the evolution of microbes. EMBO Rep. 2001, 2, 376-381.

25. Ochman, H. Lateral and oblique gene transfer. Curr. Opin. Genet. Dev. 2001, 11, 616-619.

26. Gogarten, J.P.; Doolittle, W.F.; Lawrence, J.G. Prokaryotic evolution in light of gene transfer. Mol. Biol. Evol. 2002, 19, 2226-2238.

27. Lan, R.; Reeves, P.R. When does a clone deserve a name? A perspective on bacterial species based on population genetics. Trends Microbiol. 2001, 9, 419-424.

28. Nakamura, Y.; Itoh, T.; Matsuda, H.; Gojobori, T. Biased biological functions of horizontally transferred genes in prokaryotic genomes. Nat. Genet. 2004, 36, 760-766.

29. Hacker, J.; Kaper, J.B. Pathogenicity islands and the evolution of microbes. Annu. Rev. Microbiol. 2000, 54, 641-679.

30. Hanahan, D. Studies on transformation of Escherichia coli with plasmids. J. Mol. Biol. 1983, 166, 557-580.

31. Uematsu, T.; Takatsu, A.; Ohata, K. A medium for the selective isolation of Pseudomonas cichorii. Ann. Phytopathol. Soc. Jpn. 1982, 48, 425-432.

32. Sambrook, J.; Fritsch, E.F.; Maniatis, T. Molecular Cloning: A Laboratory Manual, 2nd ed.; Cold Spring Habor Laboratory Press: Cold Spring Habor, NY, USA, 1989.

33. Tsuge, S.; Furutani, A.; Fukunaka, R.; Kubo, Y.; Horino, O. Growth complementation of hrpXo mutants of Xanthomonas oryzae pv. oryzae by virulent strains in rice cultivars resistant and susceptible to the parental strain. J. Gen. Plant Pathol. 2001, 67, 51-57. 
34. Gay, P.; Le Coq, D.; Steinmetz, M.; Berkelman, T.; Kado, C.I. Positive selection procedure for entrapment of insertion sequence elements in gram-negative bacteria. J. Bacteriol. 1985, 164, 918-921.

35. Kanda, A.; Yasukohchi, M.; Ohnishi, K.; Kiba, A.; Okuno, T.; Hikichi, Y. Ectopic expression of Ralstonia solanacearum effector protein PopA early in invasion results in loss of virulence. Mol. Plant Microbe Interact. 2003, 16, 447-455.

36. Staskawicz, B.; Dahlbeck, D.; Keen, N.; Napoli, C. Molecular characterization of cloned avirulence genes from race 0 and race 1 of Pseudomonas syringae pv. glycinea. J. Bacteriol. 1987, 169, 5789-5794.

37. de Feyter, R.; Gabriel, D.W. Use of cloned DNA methylase genes to increase the frequency of transfer of foreign genes into Xanthomonas campestris pv. malvacearum. J. Bacteriol. 1991, 173, 6421-6427.

38. Sukchawalit, R.; Vattanaviboon, P.; Sallabhan, R.; Mongkolsuk, S. Construction and characterization of regulated L-arabinose-inducible broad host range expression vectors in Xanthomonas. FEMS Microbiol. Lett. 1999, 181, 217-223.

39. Kimura, M. A simple method for estimating evolutionary rates of base substitutions through comparative studies of nucleotide sequences. J. Mol. Evol. 1980, 16, 1111-1120.

\section{Supplementary Material}

\section{Construction of Plasmids for aldH and pat-Deletion Mutants}

To construct a plasmid, pD4-1-2KmSacB, for creation of an ald $H$-deleted mutant ( $\triangle$ aldH) from SPC9018, a 780-bp fragment, designated D4-1, was first amplified by PCR from the genomic DNA of SPC9018 with the primers Kpn-D4-1-Fw and Bam-D4-1-Rv (Figure S1). The BamHI and KpnI-digested D4-1 was ligated into BamHI and KpnI sites of pHSG398 and the resulting plasmid was designated pD4-1. A 1,080-bp fragment, designated D4-2, was PCR-amplified from the genomic DNA of SPC9018 with the primers Bam-D4-2-Fw and Sal-D4-2-Rv. The SalI- and BamHI-digested D4-2 was ligated into the SalI and BamHI sites of pD4-1 and the resulting plasmid was designated pD4-1-2. A 1.4-kb fragment, designated Bam-Km, was PCR amplified from the DNA of pUCK191 with the primers Bam-Km1 and Bam-Km2. The BamHI-digested Bam-Km was ligated into the BamHI site of pD4-1-2 and the resulting plasmid was designated pD4-1-2Km. A 2.6-kb fragment, designated Sal-SacB, was amplified via PCR from pUCD800 DNA with the primers Sal-SacB1 and Sal-SacB2. The SalI-digested Sal-SacB was ligated into the SalI site of pD4-1-2Km and pD4-1-2KmSacB was constructed.

To construct pD5-1-2KmSacB for creation of a pat-deleted mutant ( $\Delta$ pat) from SPC9018, a 906-bp fragment, designated D5-1, was amplified by PCR from the genomic DNA of SPC9018 with the primers delta5-1-FW-Kpn and delta5-1-RV-Bam (Figure S1). The BamHI and KpnI-digested D5-1 was ligated into the BamHI and KpnI sites of pHSG398 and the resulting plasmid was designated pD5-1. A 1,949-bp fragment, designated D5-2, was PCR-amplified from the genomic DNA of SPC9018 with the primers delta5-2-Fw-Bam and delta5-2-Rv-Sal. The SalI- and BamHI-digested D5-2 was ligated into the SalI and BamHI sites of pD5-1 and the resulting plasmid was designated pD5-1-2. The 
BamHI-digested Bam-Km was ligated into the BamHI site of pD5-1-2 and the resulting plasmid was designated pD5-1-2Km. The SalI-digested Sal-SacB fragment was ligated into the SalI site of pD5-1-2Km and $\mathrm{pD} 5-1-2 \mathrm{KmSacB}$ was constructed.

Figure S1. Assembly of open reading frames of the DNA region in the flanking region of hrp of P. cichorii strain SPC9018 genomic DNA including aldH and pat and position of primers used for constructions of plasmids for the aldH-deleted mutant (red-colored) and the pat-deleted mutant (blue-colored).

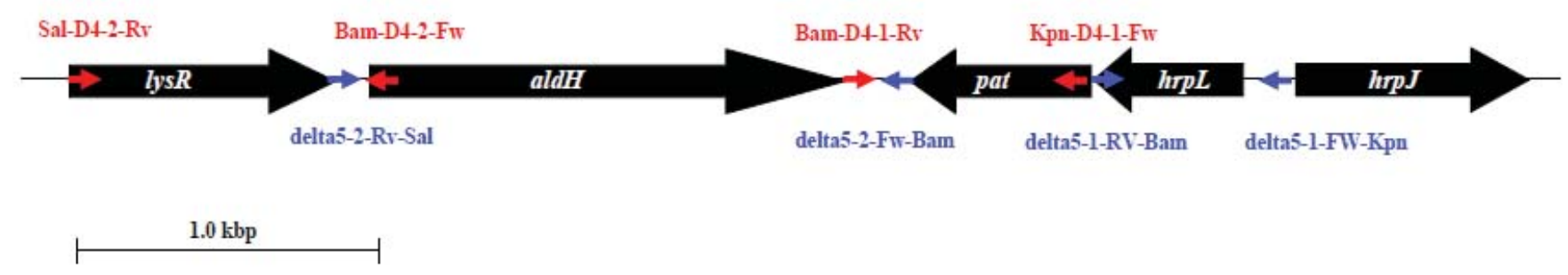

\section{Construction of Plasmids for the hrcC-Mutant}

The pUCD800 was digested with BamHI and PstI resulting in a 2.6-kb DNA fragment containing $s a c B$ that was ligated into the BamHI and PstI sites of pHSG398 to create pHSG398sacB. The plasmid, phrpFoperon [6], containing the $h r p F$ operon was digested with BamHI. The resulting 2.0-kb DNA fragment was ligated into the BamHI site of pHSG398sacB and the new plasmid named pHSG398sacBhrcC. To create a $h r c C$-mutation in pHSG398sacBhrcC, the EZ:TNTM Transposome ${ }^{\mathrm{TM}}$ system (Epicentre) was used according to the manufacturer's instructions. Transposon inserted sites in the resulting plasmids were analyzed using nucleotide sequence analysis according to the manufacturer's instructions and a plasmid, pHSG398sacBhrcCKm, into which the transposon was inserted into $h r c C$ was selected.

\section{Construction of Plasmids for Complementation of $\Delta$ aldH with aldH Originating from Pseudomonads}

A 2.0-kb DNA fragment, designated Pc-aldH, containing the predicted promoter region and the open reading frame of aldH from SPC9018, was amplified by PCR from the genomic DNA of SPC9018 with the primers Kpn-Pc-aldH-Fw and Kpn-Pc-aldH-Rv. The KpnI-digested Pc-aldH was ligated into the KpnI site of pUFR043 to create paldH. The pHSG398 was digested with Sau3AI and the resulting 1.1-kb fragment, including a chloramphenicol-resistance gene, was ligated into BamHI-site of pUC118 resulting in pUC118-cml. A 1.1-kb fragment from SacI-digested pUC118-cml was ligated into the SacI-site of paldH, thereby creating pPc-aldH.

A 2.8-kb DNA fragment, designated $\mathrm{Pv}$-aldH, containing the predicted promoter region and the open reading frame of ald $H$ from Pv9504, was PCR-amplified from the genomic DNA of Pv9504 with the primers Bam-PV4-Fw and Bam-PV4-Rv. The BamHI-digested Pv-aldH was ligated into the BamHI site of pLAFR3, creating pPv-aldH.

A 2.0-kb DNA fragment, designated Pst-aldH, containing the predicted promoter region and the open reading frame of aldH from $P$. syringae pv. tomato strain DC3000, was PCR-amplified from the 
genomic DNA of P. syringae pv. tomato strain DC3000 with the primers Kpn-Pst-Fw and Kpn-Pst-Rv. The KpnI-digested Pst-aldH was ligated into the KpnI site of pUFR043 to create pPst. A 1.1-kb fragment from SacI-digested pUC118-cml was ligated into the SacI-site of pPst, creating pPst-aldH.

A 2.4-kb DNA fragment, designated Pa-aldH, containing the predicted promoter region and the open reading frame of ald $H$ from $P$. aeruginosa strain PAO1, was amplified by PCR from the genomic DNA of $P$. aeruginosa strain PAO1 with primers Pa-Fw and Pa-Rv. The XhoI-digested $2.0 \mathrm{~kb} P$ a-aldH fragment was ligated into the $X h o \mathrm{I}$ site of $\mathrm{pBbad} 22 \mathrm{~K}$ resulting in pBbad22K-aldH. The plasmid pUC118-cml was digested with KpnI and ligated into pBbad22K-aldH, creating pPa-aldH.

\section{Construction of Plasmids for Complementation of $\Delta$ pat with pat Originating from SPC9018 and Pv9504}

A 1.5-kb DNA fragment, designated Pc-pat, containing the predicted promoter region and the open reading frame of pat from SPC9018, was amplified by PCR from the genomic DNA of SPC9018 with the primers delta5-1-Fw-Kpn and Bam-D4-2-Fw. The KpnI- and BamHI-digested Pc-pat was ligated into the KpnI and BamHI sites of pUFR043 to create ppat. A 1.1-kb fragment from SacI-digested pUC118-cml was ligated into the SacI-site of ppat, thereby creating pPc-pat.

A 1.2-kb DNA fragment, designated Pv-pat, containing the predicted promoter region and the open reading frame of pat from Pv9504, was amplified from the genomic DNA of Pv9504 using PCR with the primers Kpn-PV-ORF5-RV and Bam-PV-ORF5-FW. The BamHI- and KpnI-digested Pv-pat was ligated into the BamHI site of pUFR043 to create ppvpat. A 1.1-kb fragment from SacI-digested pUC118-cml was ligated into the SacI-site of ppvpat, thereby creating pPv-pat.

Table S1. List of bacteria analyzed and the accession numbers of the nucleotide sequences of aldH of bacteria deposited in the DDBJ/GenBank international nucleotide sequence database.

\begin{tabular}{lll}
\hline Bacteria & DDBJ Accession Number & Reference or Source \\
\hline $\begin{array}{l}\text { P. cichorii } \\
\text { SPC9018 }\end{array}$ & AB433910 & \\
MAFF730054 & AB530808 & {$[6]$} \\
SPC9037 & AB530809 & This study \\
PCL2001 & AB530810 & This study \\
MAFF211387 & AB530811 & This study \\
MAFF301158 & AB530812 & This study \\
MAFF301374 & AB530813 & This study \\
MAFF302094 & AB530814 & This study \\
MAFF302698 & AB530815 & This study \\
KH5 & AB530816 & This study \\
MAFF302152 & AB530817 & This study \\
$\boldsymbol{P}$. viridiflava & & This study \\
Pv9504 & AB530818 & \\
\hline
\end{tabular}


Table S2. Virulence of $P$. cichorii strains on the Asteraceae plants and the accession numbers of the nucleotide sequences of $n d f H$ and $r b c L$ deposited in the DDBJ/GenBank international nucleotide sequence database.

\begin{tabular}{|c|c|c|c|c|c|c|}
\hline \multirow{2}{*}{ Plant Species } & \multicolumn{4}{|c|}{ P. cichorii Strains } & \multicolumn{2}{|c|}{ DDBJ Accession Number } \\
\hline & SPC9018 & SPC9018-hrcC & $\Delta$ aldH & $\Delta$ pat & $n d h F$ & $r b c L$ \\
\hline Bidens laevis & $\mathrm{V}$ & $\mathrm{V}$ & $\mathrm{V}$ & $\mathrm{V}$ & AB530917 & AB530951 \\
\hline Bellis perennis & $\mathrm{V}$ & $\mathrm{NV}$ & $\mathrm{NV}$ & $\mathrm{NV}$ & AB530918 & AB530952 \\
\hline Calendula officinalis & $\mathrm{V}$ & $\mathrm{V}$ & $\mathrm{NV}$ & $\mathrm{NV}$ & AB530919 & AB530953 \\
\hline Callistephus chinensis & $\mathrm{V}$ & $\mathrm{V}$ & $\mathrm{V}$ & $\mathrm{V}$ & AB530920 & AB530954 \\
\hline Centaurea cyanus & $\mathrm{V}$ & $\mathrm{V}$ & NV & $\mathrm{V}$ & AB530921 & AB530955 \\
\hline Centaurea macrocephala & $\mathrm{V}$ & $\mathrm{V}$ & $\mathrm{V}$ & $\mathrm{V}$ & AB530922 & AB530956 \\
\hline Leucanthemum vulgare & $\mathrm{V}$ & $\mathrm{V}$ & NV & $\mathrm{V}$ & AB530923 & AB530957 \\
\hline Leucanthemum maximum & $\mathrm{V}$ & $\mathrm{NV}$ & $\mathrm{NV}$ & $\mathrm{NV}$ & AB530924 & AB530958 \\
\hline Coreopsis tinctoria & $\mathrm{V}$ & NV & NV & $\mathrm{V}$ & AB530925 & AB530959 \\
\hline Cosmos bipinnatus & $\mathrm{V}$ & $\mathrm{V}$ & $\mathrm{V}$ & $\mathrm{V}$ & AB530926 & AB530960 \\
\hline Crepis rubra & $\mathrm{V}$ & $\mathrm{NV}$ & NV & $\mathrm{NV}$ & AB530927 & AB530961 \\
\hline Dahlia pinnata & $\mathrm{V}$ & $\mathrm{NV}$ & $\mathrm{NV}$ & $\mathrm{V}$ & AB530928 & AB530962 \\
\hline Echinacea purpurea & $\mathrm{V}$ & $\mathrm{NV}$ & $\mathrm{NV}$ & $\mathrm{NV}$ & AB530929 & AB530963 \\
\hline Echinops ritro & $\mathrm{V}$ & $\mathrm{V}$ & $\mathrm{NV}$ & $\mathrm{V}$ & AB530930 & AB530964 \\
\hline Felicia heterophylla & $\mathrm{V}$ & $\mathrm{V}$ & $\mathrm{V}$ & $\mathrm{V}$ & AB530931 & AB530965 \\
\hline Gaillardia aristata & $\mathrm{V}$ & $\mathrm{NV}$ & NV & $\mathrm{V}$ & AB530932 & AB530966 \\
\hline Gazania splendens & $\mathrm{V}$ & $\mathrm{V}$ & NV & $\mathrm{V}$ & AB530933 & AB530967 \\
\hline Helianthus annuus & $\mathrm{V}$ & $\mathrm{V}$ & $\mathrm{V}$ & $\mathrm{V}$ & AB530934 & AB530968 \\
\hline Xerochrysum bracteatum & $\mathrm{V}$ & $\mathrm{V}$ & NV & $\mathrm{V}$ & AB530935 & AB530969 \\
\hline Leucanthemum paludosum & $\mathrm{V}$ & $\mathrm{V}$ & $\mathrm{V}$ & $\mathrm{V}$ & AB530937 & AB530971 \\
\hline Leontopodium alpinum & $\mathrm{V}$ & $\mathrm{V}$ & $\mathrm{V}$ & $\mathrm{V}$ & AB530938 & AB530972 \\
\hline Rudbeckia hirta & $\mathrm{V}$ & $\mathrm{NV}$ & NV & $\mathrm{V}$ & AB530939 & AB530973 \\
\hline Jacobaea maritima & $\mathrm{V}$ & $\mathrm{V}$ & $\mathrm{V}$ & $\mathrm{V}$ & AB530940 & AB530974 \\
\hline Pericallis cruenta & $\mathrm{V}$ & $\mathrm{V}$ & $\mathrm{NV}$ & $\mathrm{V}$ & AB530941 & AB530975 \\
\hline Tagetes patula & $\mathrm{V}$ & $\mathrm{V}$ & $\mathrm{V}$ & $\mathrm{V}$ & AB530942 & AB530976 \\
\hline Zinnia violacea & $\mathrm{V}$ & $\mathrm{V}$ & $\mathrm{V}$ & $\mathrm{V}$ & AB530943 & AB530977 \\
\hline Arctium lappa & $\mathrm{V}$ & $\mathrm{V}$ & $\mathrm{V}$ & $\mathrm{V}$ & AB530944 & AB530978 \\
\hline Chrysanthemum coronarium & $\mathrm{V}$ & $\mathrm{V}$ & $\mathrm{V}$ & $\mathrm{V}$ & AB530945 & AB530979 \\
\hline Cichorium intybus & $\mathrm{V}$ & NV & NV & NV & AB530946 & AB530980 \\
\hline Cynara scolymus & $\mathrm{V}$ & $\mathrm{V}$ & $\mathrm{V}$ & $\mathrm{V}$ & AB530947 & AB530981 \\
\hline Lactuca sativa & $\mathrm{V}$ & $\mathrm{V}$ & $\mathrm{V}$ & $\mathrm{V}$ & AB530948 & AB530982 \\
\hline
\end{tabular}

$\mathrm{V}$ : the strain was virulent to the species; NV: the strain was not virulent to the species.

Table S3. Strains and plasmids used in this study.

Relevant Characteristics

Ref. or Source

E. coli

$\mathrm{DH} 5 \alpha$

DH5 $\alpha$-aldH

P. cichorii

SPC9018

$\triangle$ aldH

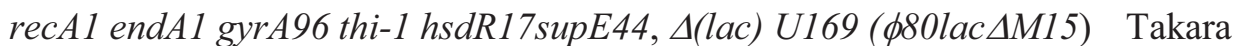

Transformant of DH5 $\alpha$ with pUC118-aldH, Ap ${ }^{\mathrm{r}}$

This study

Wild-type

[5]

aldH-deleted mutant of SPC $9018, \mathrm{Km}^{\mathrm{r}}$
This study 
Table S3. Cont.

\begin{tabular}{|c|c|c|}
\hline & Relevant Characteristics & Ref. or Source \\
\hline$\Delta$ aldH (Pc-aldH) & Transformant of $\Delta$ aldH with pPc-aldH, $\mathrm{Km}^{\mathrm{r}}, \mathrm{Cm}^{\mathrm{r}}$ & This study \\
\hline$\Delta$ aldH (Pv-aldH) & Transformant of $\Delta$ aldH with $\mathrm{pPv}$-aldH, $\mathrm{Km}^{\mathrm{r}}, \mathrm{Tc}^{\mathrm{r}}$ & This study \\
\hline$\Delta$ aldH (Pst-aldH) & Transformant of $\Delta$ aldH with pPst-aldH, $\mathrm{Km}^{\mathrm{r}}, \mathrm{Cm}^{\mathrm{r}}$ & This study \\
\hline$\Delta$ aldH (Pa-aldH) & Transformant of $\Delta$ aldH with pPa-aldH, $\mathrm{Km}^{\mathrm{r}}, \mathrm{Cm}^{\mathrm{r}}$ & This study \\
\hline$\Delta$ pat & pat-deleted mutant of SPC $9018, \mathrm{Km}^{\mathrm{r}}$ & This study \\
\hline$\Delta$ pat (Pc-pat) & Transformant of $\Delta$ pat with pPc-pat, $\mathrm{Km}^{\mathrm{r}}, \mathrm{Cm}^{\mathrm{r}}$ & This study \\
\hline$\Delta$ pat (Pv-pat) & Transformant of $\Delta$ pat with pPv-pat, $\mathrm{Km}^{\mathrm{r}}, \mathrm{Cm}^{\mathrm{r}}$ & This study \\
\hline SPC9018-hrcC & $h r c C$-deleted mutant of SPC $9018, \mathrm{Km}^{\mathrm{r}}$ & This study \\
\hline SPC9018-L & hrpL-deleted mutant of SPC $9018, \mathrm{Km}^{\mathrm{r}}$ & {$[6]$} \\
\hline \multicolumn{3}{|l|}{$P$. viridiflava } \\
\hline Pv9504 & BS type & {$[6]$} \\
\hline \multicolumn{3}{|c|}{ P. syringae pv. tomato } \\
\hline DC3000 & & {$[11]$} \\
\hline \multicolumn{3}{|l|}{ P. aeruginosa } \\
\hline PAO1 & & {$[13]$} \\
\hline \multicolumn{3}{|l|}{ Plasmid } \\
\hline pUC118 & $A m p^{r}$ & Takara \\
\hline pHSG398 & $\mathrm{Cm}^{\mathrm{r}}$ & Takara \\
\hline pLAFR3 & pLAFR1 containing HaeII fragment of pUC8 & {$[36]$} \\
\hline pUFR043 & Cosmid derivative of pUFRO42, IncW, Mob' lacZ $\alpha, \mathrm{Gm}^{\mathrm{r}}, \mathrm{Km}^{\mathrm{r}}$ & {$[37]$} \\
\hline pBbad22K & Derivative of pBAD22, mob, rep, araC, $\mathrm{Km}^{\mathrm{r}}$ & {$[38]$} \\
\hline pUCK191 & pUC18 derivative containing $\mathrm{Km}^{\mathrm{r}}$ from $\mathrm{Tn} 903$ & {$[33]$} \\
\hline pUCD800 & pUCD5 derivative containing $\operatorname{sac} B, \mathrm{Km}^{\mathrm{r}}$ & {$[34]$} \\
\hline phrpFoperon & $\begin{array}{l}\text { A } 3.6 \mathrm{~kb} \text { PCR fragment containing the } h r p F \text { operon from SPC } 9018 \\
\text { genomic DNA }\end{array}$ & [6] \\
\hline pUC118-cml & A $1.1 \mathrm{~kb}$ Sau3AI-digested pHSG398 in pUC118, $\mathrm{Ap}^{\mathrm{r}}, \mathrm{Cm}^{\mathrm{r}}$ & This study \\
\hline pPc-aldH & $\begin{array}{l}\text { A } 2.0 \mathrm{~kb} \text { PCR-fragment containing aldH of SPC } 9018 \text { and } \\
\text { SacI-digested } 1.1 \mathrm{~kb} \text { fragment of pUC118-cml in pUFR } 043\end{array}$ & This study \\
\hline pPv-aldH & A $2.8 \mathrm{~kb}$ PCR-fragment containing aldH of Pv9504 in pLAFR3 & This study \\
\hline pPst-aldH & $\begin{array}{l}\text { A } 2.0 \mathrm{~kb} \text { PCR-fragment containing aldH of DC } 3000 \text { and } \\
\text { SacI-digested } 1.1 \mathrm{kbp} \text { fragment of pUC } 118 \text {-cml in pUFR } 043\end{array}$ & This study \\
\hline pPa-aldH & $\begin{array}{l}\text { A } 2.4 \mathrm{~kb} \text { PCR-fragment containing aldH of PAO1 and KpnI-digested } \\
1.1 \mathrm{~kb} \text { fragment of pUC118-cml in pBbad22K }\end{array}$ & This study \\
\hline pPc-pat & $\begin{array}{l}\text { A } 1.5 \mathrm{~kb} \text { PCR-fragment containing pat of SPC } 9018 \text { and SacI-digested } \\
1.1 \mathrm{~kb} \text { fragment of pUC } 118 \text {-cml in pUFR } 043\end{array}$ & This study \\
\hline pPv-pat & $\begin{array}{l}\text { A } 1.2 \mathrm{~kb} \text { PCR-fragment containing pat of Pv9504 and SacI-digested } \\
1.1 \mathrm{~kb} \text { fragment of pUC118-cml in pUFR043 }\end{array}$ & This study \\
\hline
\end{tabular}

Table S4. List of primers used in this study.

\begin{tabular}{ll}
\hline Name & Sequence $^{\text {a }}$ \\
\hline Kpn-D4-1-Fw & 5'-GGGGTACCACAGTTTTGTCCCTAAACCCG-3' \\
Bam-D4-1-Rv & 5'-CGGGATCCGGCGTCCACAAAAAAGAGCG-3' \\
Bam-D4-2-Fw & 5'-CGGGATCCTCACATCGGTATCTCCTGTTG-3' \\
Sal-D4-2-Rv & 5'-GCGTCGACGCTATGATCATTCATCCTCAGC-3' \\
\hline
\end{tabular}


Table S4. Cont.

\begin{tabular}{ll}
\hline Name & 'sequence \\
\hline Bam-Km1 & 5'-CGGGATCCGGTACCCCCCCGCGCCTGATGC-3' \\
Bam-Km2 & 5'-CGGGATCCCCCACGTCTAAGAAACC-3' \\
Sal-SacB1 & 5'-CGACGCGTCGACGGATCCTTTTTAACCCATC-3' \\
Sal-SacB2 & 5'-CGACGTCGACTGCAGTTCACTTACACCGC-3' \\
delta5-1-FW-Kpn & 5'-GGGGTACCTGCCATCTGATGCTTTGAAAG-3' \\
delta5-1-RV-Bam & 5'-CGGGATCCTCACATCGCTTCGAGATCGTCTTCAG-3' \\
delata5-2-Fw-Bam & 5'-CGGGATCCTTCGCCAGCGTTGAAAAAAGGG-3' \\
delata5-2-Rv-Sal & 5'-GCGTCGACGCGATTCGTTCCTGCCGCTATC-3' \\
Kpn-Pc-aldH-Fw & 5'-GGGGTACCCCCGCATCAAACCGGTCATGG-3' \\
Kpn-Pc-aldH-Rv & 5'-GGGGTACCGTCAGACGATAGGCTGGTC-3' \\
Bam-PV4-Fw & 5'-CGGGATCCAAGCTATGATTAATCATCCAC-3' \\
Bam-PV4-Rv & 5'-CGGGATCCTCAAGCGATCGGCTGATCACTC-3' \\
Kpn-Pst-Fw & 5'-GGGGTACCCCCGCATCAAGCCGGTGATGG-3' \\
Kpn-Pst-Rv & 5'-GGGGTACCTCACGCGACAGGCTGATC-3' \\
Pa-Fw & 5'-GCTACGCGCCTGCTGCTACGGGC-3' \\
Pa-Rv & 5'-GACCGCCTACGCCGCTGCCGCAG-3' \\
Kpn-PV-ORF5-RV & 5'-GGGGTACCTCAGACAGCCTCCGATACGTG-3' \\
Bam-PV-ORF5-FW & 5'-CGGGATCCGGTGGCATCACAACTGCGTATC-3' \\
SEMI-Back & 5'-CTCACCGTTGACCAGACGC-3' \\
SEMI-Front & 5'-GTCCAGCACTTGCTGGAGC-3' \\
ORF5-RT-Fw & 5'-GGGGCCAACTCGCCGGTTAC-3' \\
aldH-Fw1 & 5'-GCGATTCGTTCCTGCCGCTATC-3' \\
aldH-Rv & 5'-CCGCTCTTTTTTGTGGACGCCGG-3' \\
16S-rRNA-Rv & 5'-AAATTCCACCACCCTCTGC-3' \\
16S-rRNA-Fw & 5'-GCCTAGGTCGGATTAGCTAG-3' \\
ndhF-11FW & 5'-GGGYTGGGACTTCTTCTTTTYCC-3' \\
ndhF-22RV & 5'-CCSCCKACYSATTTAATAACC-3' \\
1-1 & 5'-ATGTCACCACAAACAGAGACTAAAGC-3' \\
NN3-2 & 5'-GCAGCAGCTAGTTCCGGGCTCCA-3' \\
ndhF-11RV & 5'-TAGGYGAATACAACCAACTATC-3' \\
ndhF-22FW & 5'-TTGCYTGTTTTTGGTCNAAAGATG-3' \\
1-2FW & 5'-CAGTACTTCCATGTTGG-3' \\
1-2RV & 5'-TATCCAACAAGAGTTTCC-3' \\
\hline & 'Restiction enzyme \\
\hline
\end{tabular}

${ }^{a}$ Restriction enzyme sites in the primer sequence are underlined: BamHI GGATCC, EcoRI $\underline{\text { GAATTC, }}$ KpnI GGTACC, and SalI GTCGAC.

(C) 2012 by the authors; licensee MDPI, Basel, Switzerland. This article is an open access article distributed under the terms and conditions of the Creative Commons Attribution license (http://creativecommons.org/licenses/by/3.0/). 